\title{
Applications for tracking reading behavior on the Macintosh
}

\author{
SUSAN R. GOLDMAN and ELIZABETH U. SAUL \\ Vanderbilt University, Nashville, Tennessee
}

\begin{abstract}
Two applications for the Macintosh that permit students to read the sentences in a text in any order also provide a record of reading behavior from which processing time and reading strategies can be determined. The applications differ: READIT! presents the sentences one at a time, whereas SELECT THE TEXT presents the entire passage with a mask, which resembles the moving window technique. Unique to these applications is that students may return to sentences to reread them any number of times and in any order. Because these applications allow students to reexamine parts of the text, the reading that they enable is more similar to normal reading than has been the case with previous methodologies for tracking student reading behavior. The applications are described, and a summary of the major results of the work in which we have used the applications is provided.
\end{abstract}

For some time, we have been conducting research on the strategies that students use when they are learning new information from text and textbook-like passages (e.g., Goldman \& Durán, 1988). Our specific interest has been to identify different kinds of student reading strategies and to understand how various text variables affect them (Goldman, 1988a, 1988b; Goldman \& Saul, 1990a). To do so, it is important to keep track of exactly what students are reading and how long they are spending on it. Although there are a number of existing techniques designed to provide this information, they are subject to the criticism that they distort the normal reading process by presenting words or sentences one at a time and by restricting readers to unidirectional movement through the text (see, e.g., Aaronson \& Scarborough, 1976; Lorch $\&$ Chen, 1986). For example, the moving window technique in which students read one word at a time and cannot see any of the surrounding words does not permit students to go back and reread material (Graesser \& Riha, 1984; Haberlandt, 1984; Haberlandt \& Graesser, 1985; Just, Carpenter, \& Woolley, 1982). Similarly, singlesentence text-presentation programs permit readers to see each sentence in the text successively for a single viewing (see, e.g., Lorch \& Chen, 1986). However, eyemovement data collected by Buswell (1937) and more recently by Erlich and Raynor (1981) indicate that readers do reread if the methodology permits them to do so, sug-

This work was supported, in part, by the Cognitive Science Program, Office of Naval Research, under Contract N00014-85-K-0562, Authorization Number NR442c015. The current paper is a modified version of a poster, "Tracing Reading Strategies on the Macintosh," which was presented as part of the poster symposium "Using Technology to Enhance Literacy,"' held at the American Educational Research Association meeting, Boston, MA, April 1990. Address correspondence concerning this work, or requests for copies of the technical manuals and software, to Susan R. Goldman, Box 45 Peabody, Vanderbilt University, Nashville, TN 37203 . E-mail: GoldmanS@VUCTRVAX.bitnet. gesting the need for a more flexible text-presentation methodology.

We have developed a methodology that permits students to read the sentences in a text in any order, yet provides a record of reading behavior from which processing time and reading strategies can be determined. On the basis of traces of the reading behavior, we have identified several different reading strategies. The methodology relies on two applications for the Macintosh: READIT! (Saul, Pohl, \& Goldman, 1988) and SELECT THE TEXT (Gontier, Saul, \& Goldman, 1989). Our purpose in this paper is twofold: (1) to describe two Macintosh applications that permit the assessment of reading strategies under relatively normal reading conditions; and (2) to provide a brief summary of results which show that most individuals demonstrate strategy flexibility of a sort that is masked by more restrictive methodologies.

\section{Applications for Assessing Reading Strategies}

Two features are common to the two Macintosh applications that we have developed to assess reading strategies. (1) Readers control the presentation rate and the presentation order of text segments. (2) The applications create an output file for each text, which includes the sequence in which text segments are read and the amount of time the reader spends on a segment each time the reader examines it. From this output file, we can construct a trace of the reading behavior, as well as compute summary statistics.

\section{READIT!}

Technical information. READIT! is written in Pascal. It can be used on any Macintosh with System 4.0 or higher. A segment may not exceed 684 characters (including blanks); a passage may not contain more than 30 segments; and a test file may contain no more than 35 passages. READIT! has specific look-up addresses for 
texts to be used as input, and it has sites for output files. Timing for each segment is accurate to 1/60th of a second, corresponding to one Macintosh "tick." Times are accurately kept to the third decimal place and are automatically rounded. Our "segments" were all defined as sentences, but it is equally possible to make segments of one letter, one word, or a phrase. Alphanumeric mixtures are also possible in READIT!. Screen saver software, such as Moire or Pyro, is known to cause READIT! to crash and should be deactivated first.

Overview of the application. The READIT! application presents sentences one at a time, as shown in Figure 1. Readers move forward or backward through the text by pressing the designated keys. Readers can spend as much time as they want on each sentence, and they can go backward and forward through the sentences as often as they wish. A hard-copy printed trace of the reader's behavior can be output. In addition, the application includes datareduction routines that summarize reading-rate and processing-time data for each sentence. These data are "per word," to adjust for differences in the number of words in different sentences. Short descriptions of each of the modules in the program will be provided here. Additional details and instructions for running the application are provided in the technical manual (Saul et al., 1988).

The application is structured into five modules, named more or less mnemonically. It requires as input text-only files that have been created with any word processing program. When the text files are created, segments corresponding to the units to be presented on the screen are delimited with a symbol chosen by the user. In our work, we chose the sentence as the segment and the symbol "." as the delimiter. The symbol does not show up on the screen when the segment is displayed.

Once the delimited text-only passages have been created, the module Buildfile is used to create a presentation file, or testfile, for a specific subject. The user is prompted for the order in which the passages are to be presented and the testfile is created. The testfile may contain up to 35 passages. Within each passage, the delimited segments are numbered sequentially, from 1 to $n$. The testfile created by Buildfile serves as input to the second module, Readit, which is the actual presentation module.

The Readit module presents to subjects the contents of testfiles created by Buildfile and produces output files containing information regarding the sequence in which segments were read and the amount of time a segment was displayed on each of its presentations. One segment at a time is displayed on the screen, and subjects control the display time using three keys - "control" (previous segment), " 0 " on the numeric keypad (next segment), and "enter" on the numeric keypad (finished). There is no limitation on the number of times a segment can be displayed; however, each segment must be displayed at least once before the "finish" command may be entered. When the next passage begins, subjects cannot return to a previous passage. Readit creates three output files per testfile: one contains the time spent on each segment each time it is read, a second contains the order in which the segments are read, and the third contains the actual segments.

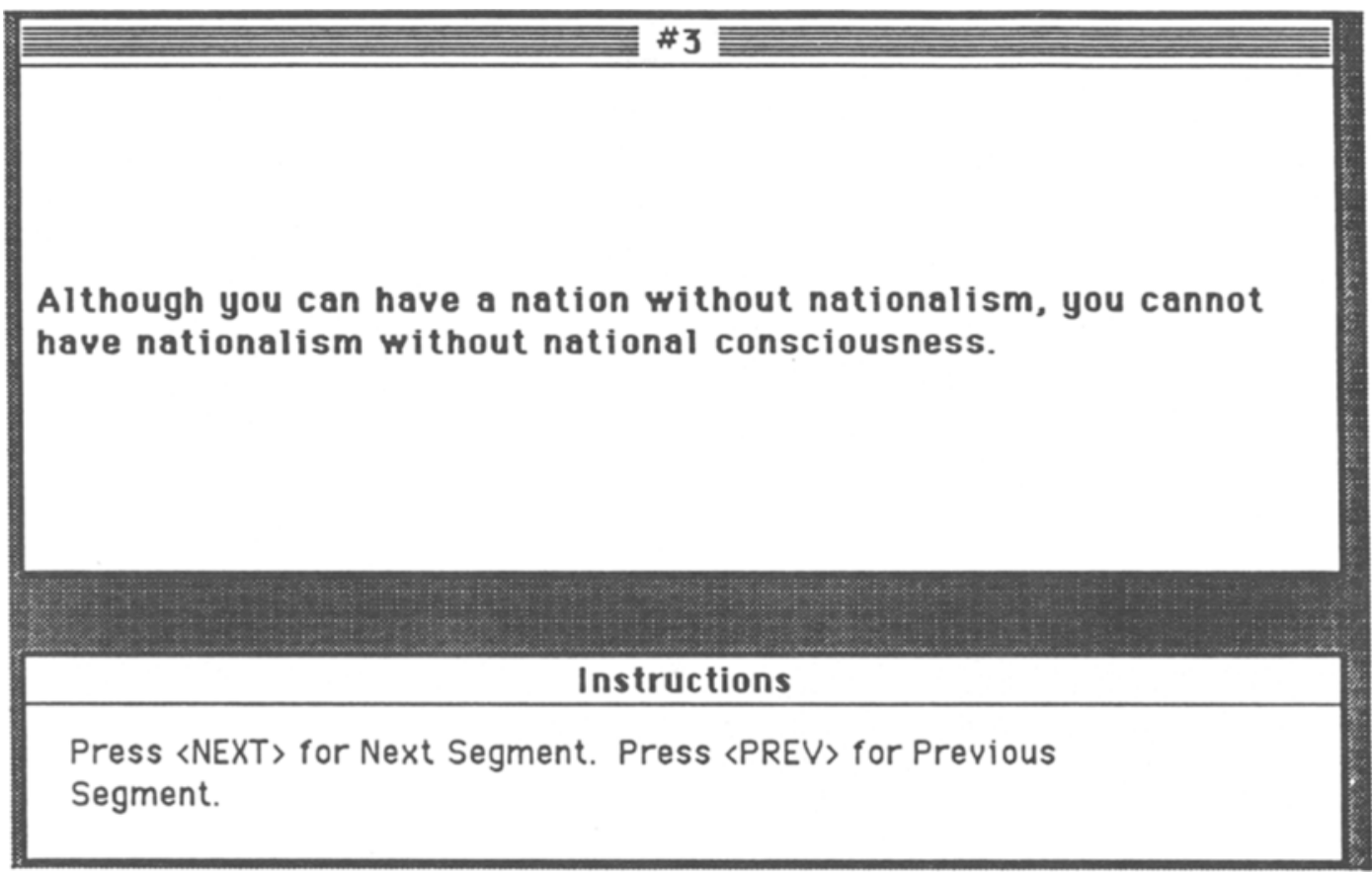

Figure 1. Sample screen from the READIT! application. 
With the Viewdata module, each of these files can be printed (screen or hard copy). However, Viewdata does not permit a file to be edited.

The Convert module takes the information from the time and segment-order output files, combines them into a sequenced list, and does some simple calculations on the time data. The calculations include the number of words and number of letters in the segment; the number of seconds per segment, per word, and per letter; the cumulative time per segment, word, and letter (relevant if a segment is read more than once); and the total time for the passage. We have found it useful to edit the files produced by Convert for viewing times that are too short to reflect "true" reading. In our work, we set a criterion of $0.7 \mathrm{sec}$, which is based on pilot studies in which expert readers pressed the "next" key as quickly as the text came up on the screen. Segments viewed for $0.7 \mathrm{sec}$ or less were judged to be "skimmed over" and were removed from the trace file. The editing can be done via a word processing program operating on the file produced by Convert; the edited file should be saved in the text-only format. From the edited file, we constructed time graphs for each passage. A sample time graph and the file from which it was drawn are shown in Figure 2. Heavy lines denote reading, and light ones skimming. Arrows indicate the direction of reading, and filled circles indicate a single sentence that was skimmed to, read, and skimmed away from.

The module Lastone (because it is the final step) performs summarizing computations on the information in the file produced by the Convert module. Lastone summarizes the time information for each segment and prints the segments in their canonical order. Sample output is provided in Table 1. Lastone summarizes the (1) number of times the segment is viewed, (2) number of words in the segment, (3) total number of words read per segment (i.e., [1] $\times$ [2]), (4) total time spent reading the segment, (5) reading rate (i.e., [4]/[3]), and (6) process time per segment (i.e., [4]/[2]). Also reported are passage-level statistics, such as total words in the passage, total words read, rate per passage, and process time per passage.

In summary, READIT! permits students to control the rate and presentation order of text segments, which appear one at a time on the screen. Experimenters can segment the text in any manner desired. The program collects the time and segment-reading-order information that can be edited by the experimenter before a final summarization table is produced.

\section{SELECT THE TEXT}

Technical information. SELECT THE TEXT is written in Pascal. It can be used on any Macintosh with System 4.1 or higher. A hard drive will make the application run much faster, but it can be run from diskettes. At present, there are no known length limitations to the allowable number of letters per word, words per sentence, sentences per passage, or number of passages per test file. The program segments texts according to the occurrence of three specific punctuation marks found in English: the period, the exclamation point, and the question mark. Like READIT!, SELECT THE TEXT has specific look-up addresses for texts to be used as input, and it has sites for output files. The timing is the same as it is for READIT!.

\begin{tabular}{|c|c|c|c|c|c|c|}
\hline \multicolumn{2}{|c|}{ Output File Data } & \multicolumn{3}{|c|}{ Time Graph } & \multicolumn{2}{|c|}{ Schematic Reading Trace } \\
\hline $\begin{array}{c}\text { Sentence } \\
\text { number }\end{array}$ & $\begin{array}{l}\text { Reading } \\
\text { time }\end{array}$ & $\begin{array}{l}\text { Sentence } \\
\text { number }\end{array}$ & $\begin{array}{l}\text { Time } \\
\text { graph }\end{array}$ & & $\begin{array}{c}\text { Sentence } \\
\text { number }\end{array}$ & $\begin{array}{l}\text { Schematic } \\
\text { reading trace }\end{array}$ \\
\hline 1 & 18.450 & 1 & $18.450,6.880$ & & 1 & \\
\hline 2 & 8.550 & 2 & $8.550 / 1.200$ & & 2 & \\
\hline 1 & 6.880 & 3 & 14.583 & & 3 & \\
\hline 2 & 1.200 & 4 & 5.250 & & 4 & \\
\hline 3 & 14.583 & 5 & 17.267 & & 5 & \\
\hline 4 & 5.250 & 6 & 9.633 & 0.867 & 6 & \\
\hline 5 & 17.267 & 7 & 3.017 & 4 & 7 & \\
\hline 6 & 9.633 & 8 & 2.767 & & 8 & \\
\hline 7 & 3.017 & 9 & 3.833 & & 9 & \\
\hline 8 & 2.767 & 10 & 6.267 & 1.867 & 10 & \\
\hline 9 & 3.833 & 11 & 14.250 & 14.667 & 11 & \\
\hline 10 & 6.267 & 12 & 0.933 & & 12 & \\
\hline 11 & 14.250 & & & & & \\
\hline 12 & 0.933 & & & & & \\
\hline 11 & 14.667 & & & & & \\
\hline 10 & 1.867 & & & & & \\
\hline 6 & 0.867 & & & & & \\
\hline
\end{tabular}

Figure 2. Sample derivation of time graph and schematic reading trace from READIT! output. Heavy lines indicate reading, while light ones indicate skimming. The filled circle indicates that the single sentence was read while those around it were skimmed. Arrows indicate the reader's general direction of movement through the text. 
Table 1

Sample Output from Lastone Module of the READIT! Application THE FOLLOWING OUTPUT IS FOR SUBJECT "SUB2."

\#V ........ Number of Segment Views.

\#W ....... Number of Words in Segment.

TWR ..... Total Words Read per Segment.

RT ....... Total Reading Time per Segment.

RT/TWR . . Reading Time / Total Words Read per Seg.

$\mathrm{RT} / \# \mathrm{~W}$... . Reading Time / Number of Words in Seg.

$\begin{array}{lcccccc}\text { SEGMENT } & \# \mathrm{~V} & \# W & \text { TWR } & \text { RT } & \text { RT/TWR } & \text { RT/\#W } \\ \text { 13,3.SEG.1 } & 2 & 20 & 40 & 20.883 & 0.522 & 1.044 \\ \text { 13,3.SEG.2 } & 2 & 17 & 34 & 18.317 & 0.539 & 1.077 \\ \text { 13,3.SEG.3 } & 2 & 17 & 34 & 34.334 & 1.010 & 2.020 \\ \text { 13,3.SEG.4 } & 2 & 10 & 20 & 21.400 & 1.070 & 2.140 \\ \text { 13,3.SEG.5 } & 2 & 15 & 30 & 12.916 & 0.431 & 0.861 \\ \text { 13,3.SEG.6 } & 2 & 19 & 38 & 16.700 & 0.439 & 0.879 \\ \text { 13,3.SEG.7 } & 2 & 22 & 44 & 23.117 & 0.525 & 1.051 \\ \text { 13,3.SEG.8 } & 2 & 21 & 42 & 19.117 & 0.455 & 0.910 \\ \text { 13,3.SEG.9 } & 2 & 19 & 38 & 10.083 & 0.265 & 0.531 \\ \text { 13,3.SEG.10 } & 2 & 7 & 14 & 12.534 & 0.895 & 1.791 \\ \text { 13,3.SEG.11 } & 3 & 15 & 45 & 27.067 & 0.601 & 1.804 \\ \text { 13,3.SEG.12 } & 3 & 14 & 42 & 17.483 & 0.416 & 1.249 \\ \text { 13,3.SEG.13 } & 2 & 12 & 24 & 17.200 & 0.717 & 1.433 \\ \text { 13,3.SEG.14 } & 2 & 19 & 38 & 21.900 & 0.576 & 1.153 \\ \text { 13,3.SEG.15 } & 2 & 16 & 32 & 7.312 & 0.229 & 0.457 \\ \text { 13,3.SEG.16 } & 2 & 11 & 22 & 7.655 & 0.348 & 0.696 \\ \text { 13,3.SEG.17 } & 2 & 26 & 52 & 38.717 & 0.745 & 1.489 \\ \text { 13,3.SEG.18 } & 2 & 20 & 40 & 29.267 & 0.732 & 1.463 \\ \text { 13,3.SEG.19 } & 2 & 13 & 26 & 32.650 & 1.256 & 2.512\end{array}$

Total Words In Passage $=313$

Total Words Read $=655$

Total Reading Time $=388.652$

Total Reading Time / Total Words Read $=0.593$

Total Reading Time / Total Words In Passage $=1.242$

The output files are opened with Microsoft Word. Screen savers such as Moire and Pyro are known to crash the program, and need to be deactivated before the application is run.

There are two conventions used during passage presentation. First, if a student clicks outside of the window in which the passage is presented, the time interval is recorded and appears under "segment \#0" in the output file. Second, it is possible for the student to expose two sentences at the same time by clicking on the very end of the masking for the first sentence, where the punctuation mark lies hidden. We instruct students not to click on this area of the masking.

Overview of the application. SELECT THE TEXT presents readers with a full screen of text. All text but the sentence currently selected by the reader is masked, and the white spaces denote sentence boundaries, as illustrated in Figure 3. This application is an adaptation of the moving window technique: Readers unmask a sentence by clicking on it; when they unmask another sentence, the first sentence is remasked. Thus, only one sentence at a time is visible, but the graphic (layout) features of the text are visible to the reader. Readers can unmask sentences in any order. An active scroll bar permits texts to extend over multiple screens. Additional details and instructions for running the application are provided in the technical manual (Gontier et al., 1989).

The advantage of this application over the READIT! application is that information regarding paragraph indentation, length of the passage, and position of the sentence in the paragraph and passage are retained in the display. Thus, SELECT THE TEXT permits an examination of memory for the location of particular sentences in the passage and of the role of graphic (layout) cues such as paragraph indentation and spatial position of the sentence in the text.

There are two modules in the SELECT THE TEXT application: MakeMoveWindTest (MMWT) and MoveWind (MW). MW is the actual presentation program, and MMWT is the module in which passages are prepared for presentation, including entering the text and creating the sequences in which the passages will be presented. MMWT consists of an editor and a builder. The editor permits creation or alteration of previously created passages. It operates like a stripped-down word processing application and creates text-only files. Because of the way in which the presentation module "reads" the passages, there are several rules to follow when creating the passages. For example, MW interprets three characters as end-of-segment markers: the period (.), the question mark (?), and the exclamation point (!). If these occur in the text in places other than at the end of a segment, the user will end up with incorrectly segmented text. After the passages have been created, the builder component of MMWT permits the sequencing of the passages for presentation. After the experimenter selects the relevant passages and orders them in the appropriate way, the sequence is saved, with an experimenter-designated file name.

To access the sequence for presentation to a student, MW requests the subject number and the file name separately. Each passage in the sequence is presented in the manner indicated in Figure 3. Students click on the black mask that covers the sentence they wish to read. When they click on a different sentence mask, the exposed sentence is remasked. MW creates an output file for the student, which consists of the order in which the specific sentences were unmasked and the amount of time for which they were exposed each time they were unmasked. Students can read and reread the sentences in any order they choose. To move from one passage to the next, the student hits any key on the keyboard that is not a function key. Students cannot return to previously read passages.

Output files are automatically saved with the subject's name (as entered by the experimenter) in the file name. The output file contains raw data and a summary table, like that in Table 2. The raw data section lists the sequence in which segments were exposed and the length of time for which each was exposed. From the sequence and time data in Table 2, a time graph and schematic trace of the reading behavior can be constructed, like those shown in Figure 2. The summary data section of Table 2 provides the following information for each sentence: (1) the num- 


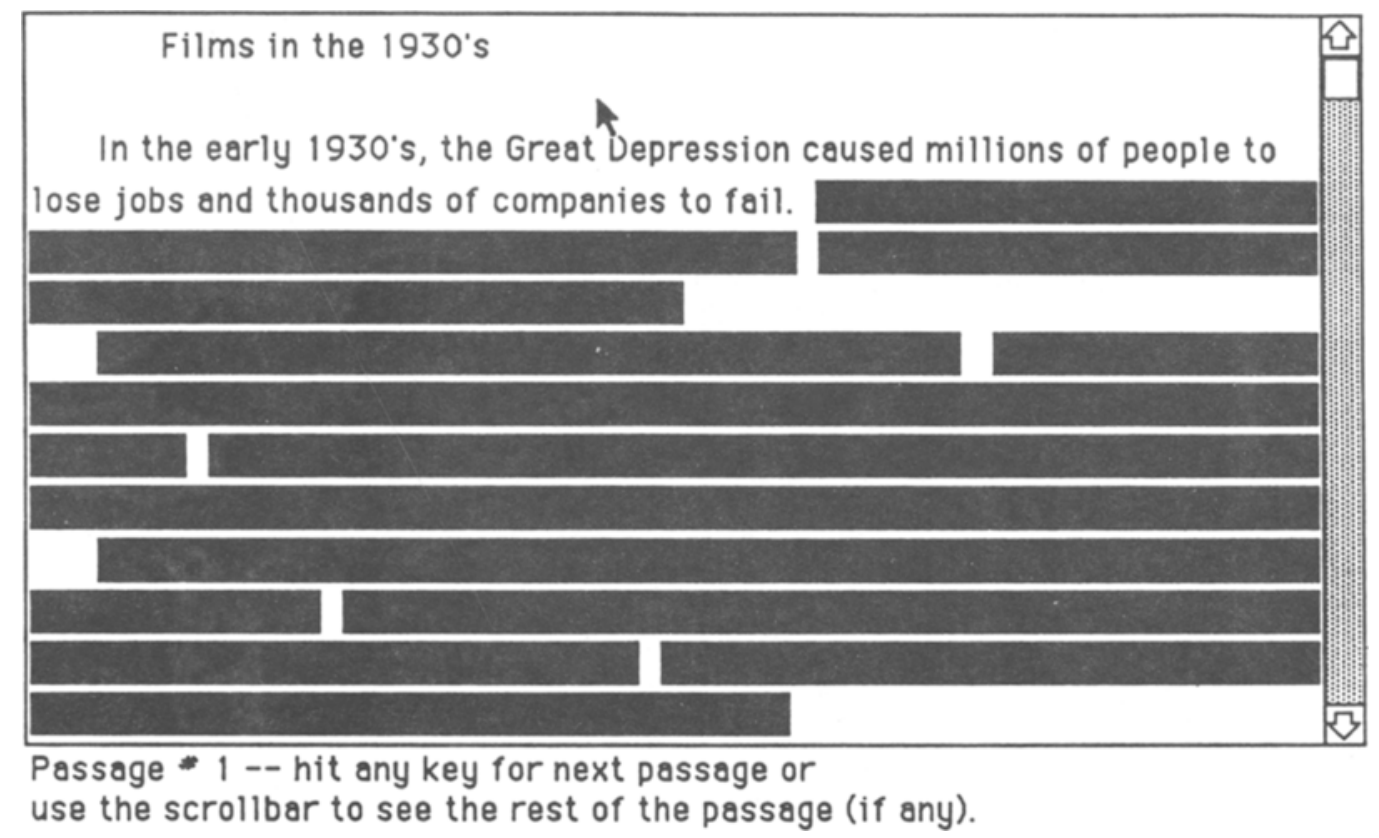

Figure 3. Sample screen from the SELECT THE TEXT application.

ber of words in the sentence; (2) the total amount of time for which the sentence was exposed (TT); (3) the rate per word (TT/[number of words in the segment] $\times$ [number of times the segment was read]); and (4) the process time per word (TT/[number of words in the segment]).

\section{Summary of Experimental Work Enabled by These Applications}

Using the Macintosh applications READIT! and SELECT THE TEXT, we have examined the strategies that college students use when they read texts like those found in introductory-level textbooks. The strategies are derived from the reading-trace outputs of the two applications. We score the traces for the presence of a number of different reading strategies-such as going back to reread a single sentence, going back to read several previous sentences, getting to the end of the passage, skimming all the way back to the beginning, and so forth. These strategies are described more fully in Goldman and Saul (1990a). By examining the types of strategies that a reader uses in a particular passage, we have been able to identify three distinct global approaches to reading: reading once through the passage; reviewing selected sentences after reading the whole thing once through; and rereading selected sentences prior to getting all the way through the passage.

In several studies, we examined the effects of manipulating surface text characteristics, such as whether or not targeted information was explicitly signaled, on reader strategies (Goldman, 1988a; Goldman \& Saul, 1990a; Goldman, Durán, Murray, Saul, \& Smith, 1989). Currently, we are comparing the effects on reader strategies of conventional paragraph placement (i.e., at the beginning of a new topic within the passage) with paragraph placement that violates convention. In this study, we are also examining the effect of different tasks (recall versus summary) on the reading strategies that students use (Goldman \& Saul, 1990b).

The data on reading strategies that we have derived from the output of READIT! and SELECT THE TEXT revealed several important findings, the details of which are reported in Goldman (1988a, 1988b), Goldman and Saul (1990a), and Goldman et al. (1989). Here, we will review the major findings from these studies very briefly.

1. There was substantial variability in how passages were read, in terms of global approaches and local rereading strategies. The majority of individuals used at least two different global approaches and at least two different local strategies.

2. The variability in local strategy use was not systematically related to the signaling manipulation.

3 . The variability was related to a variety of semantic and structural features of text, such as syntactic complexity, pronominal reference, and topic-shift detection.

4. When readers did not reread, we found that reading time per word predicted recall. However, when readers did go back and reread material, measures of strategy flexibility were more predictive of recall than were readingtime measures. Greater flexibility in strategy use was positively related to recall.

5. A strategy competition model has been proposed to explain these results, and there is preliminary validation of the model from a verbal protocol study (Goldman \& 
Table 2

Sample Output from SELECT THE TEXT application: Raw Sentence Sequence and Summary Table

SELECT THE TEXT Output for Subject \#1

Raw Sequence data:

S\# seconds

12.283

8.800

8.583

4.333

6.783

12.467

4.133

7.250

5.683

9.833

26.533

3.833

3.333

16.117

7.967

5.083

17.333

9.133

4.850

3.333

1.233

2.300

4.517

3.600

4.267

4.933

2.433

3.133

4.217

5.867

4.500

10.300

Summary data:

\begin{tabular}{rrrrr} 
S\# & words & \multicolumn{1}{c}{ TT } & PTpW & RPW \\
1 & 24 & 12.283 & 0.512 & 0.512 \\
2 & 13 & 8.800 & 0.677 & 0.677 \\
3 & 21 & 18.883 & 0.899 & 0.450 \\
4 & 8 & 8.833 & 1.104 & 0.552 \\
5 & 10 & 12.650 & 1.265 & 0.632 \\
6 & 19 & 16.683 & 0.878 & 0.439 \\
7 & 9 & 7.267 & 0.807 & 0.404 \\
8 & 8 & 7.250 & 0.906 & 0.906 \\
9 & 9 & 8.117 & 0.902 & 0.451 \\
10 & 17 & 14.767 & 0.869 & 0.434 \\
11 & 18 & 30.800 & 1.711 & 0.856 \\
12 & 12 & 7.433 & 0.619 & 0.310 \\
13 & 7 & 3.333 & 0.476 & 0.476 \\
14 & 15 & 20.633 & 1.376 & 0.688 \\
15 & 13 & 7.967 & 0.613 & 0.613 \\
16 & 10 & 7.383 & 0.738 & 0.369 \\
17 & 18 & 21.900 & 1.217 & 0.406 \\
18 & 10 & 4.850 & 0.485 & 0.485 \\
19 & 12 & 9.133 & 0.761 & 0.761 \\
\hline
\end{tabular}

Note $-S \#=$ sentence number. $\mathrm{TT}=$ total time reading, $\mathrm{PTpW}=$ process time per word, $\mathrm{RPW}=$ rate per word.
Saul, 1990a). The verbal protocols in conjunction with the reading traces are enabling us to begin to understand the factors that affect readers' choices of strategy.

\section{Conclusions}

The two applications we have developed, READIT! and SELECT THE TEXT, provide relatively unobtrusive means of examining reader strategies under conditions similar to those of normal reading, without sacrificing reading-time data. Prior research efforts may have relied too much on data gathered under artificially constrained conditions, which created more stereotypy in reading behavior than is the case in unconstrained conditions. The time graphs and reading traces made possible by these two applications have revealed that there are differential effects of various text features on reader strategies. Such information may be useful in diagnosing individual differences in sensitivity to specific text features.

The flexibility evident in the traces of individual readers and its relationship to recall indicate that it is important for readers to understand that different texts may require different reading strategies. Strategic behavior is adaptive to the specific demands and characteristics of a text. From a diagnostic perspective, readers who always use the same approach and strategy may not be reading effectively. Theories of reading comprehension and of how people learn from text provide unrealistic accounts of normal reading, if the empirical data on which they are based have prevented individuals from using the reading strategies they normally do. When the adaptability inherent in strategy flexibility is masked by experimental conditions that artificially limit readers' behaviors, readers will appear to be more unidimensional than they are under naturally occurring conditions. The applications we have developed allow us to track backward, as well as forward, movement through a text, and thus they permit us to assess reading under conditions more similar to those that obtain during normal reading.

\section{REFERENCES}

aAronson, D., \& SCarborough, H. S. (1976). Performance theories for sentence coding: Some quantitative evidence. Journal of $E x$ perimental Psychology: Human Perception \& Performance, 2, 56-70

BUSWELL, G. T. (1937). How adults read. Supplementary Educational Monographs (No. 45). Chicago: University of Chicago Press.

ERLICH, S. F., \& RAYNOR, K. (1981). Contextual effects on word perception and eye movements during reading. Journal of Verbal Learning \& Verbal Behavior, 20, 641-655.

Goldman, S. R. (1988a, April). The role of sequence markers on reading and learning strategies. Paper presented at the meeting of the American Educational Research Association, New Orleans.

Goldman, S. R. (1988b, November). Strategies for understanding in formation organization in discourse. Paper presented at the Twentyninth Annual Meeting of the Psychonomic Society, Chicago. 
Goldman, S. R., Durín, R. P. (1988). Answering questions from oceanography texts: Reader, task and text interactions. Discourse Processes, 11, 373-412.

Goldman, S. R., Durán, R. P., Murray, J., Saul, E. U., \& Smith, M. (1989). Reasoning and comprehension processes of linguistic minority persons leaming from text. Final report to the Office of Naval Research Cognitive Science Program under contract N00014-85-K-0562, authorization number NR442c015. (Defense Technical Information Center \#A211951). Santa Barbara: University of California.

Goldman, S. R., \& SAUl, E. U. (1990a). Flexibility in text processing: A strategy competition model. Learning \& Individual Differences, 2, $181-219$.

Goldman, S. R., \& SAUl, E. U. (1990b). Paragraphing and task effects on reading strategies. Paper presented at the 31st Annual Meeting of the Psychonomic Society, New Orleans.

Gontier, P., Saul, E. U., a Goldman, S. R. (1989, August). SELECT THE TEXT: A Macintosh moving window application (Tech. manual). Santa Barbara: University of California.

Graesser, A. C., RiHA, J. R. (1984). An application of multiple regression techniques to sentence reading times. In D. E. Kieras \&
M. A. Just (Eds.), New methods in reading comprehension research (pp. 183-218). Hillsdale, NJ: Erlbaum.

HABERLANDT, K. (1984). Components of sentence and word reading times. In D. E. Kieras \& M. A. Just (Eds.), New methods in reading comprehension research (pp. 219-251). Hillsdale, NJ: Erlbaum.

Haberlandt, K., \& Graesser, A. C. (1985). Component processes in text comprehension and some of their interactions. Journal of $E x$ perimental Psychology: General, 114, 357-374.

Just, M. A., CARPEnter, P. A., \& WoOlley, J. D. (1982). Paradigms and processes in reading comprehension. Journal of Experimental Psychology: General, 111, 228-238.

LORCH, R. F., CHEN, A. H. (1986). Effects of number signals on reading and recall. Journal of Educational Psychology, 78, 263-270.

Saul, E. U., Pohl, M., \& Goldman, S. R. (1988, December). READIT! A text presentation application for the Macintosh (Tech. manual). Santa Barbara: University of California.

(Manuscript received May 29, 1990; revision accepted for publication September 28, 1990.) 